\title{
Creating Language Environment at Al- Mashduqiah Islamic Education Center Patokan Kraksaan Probolinggo
}

Mamluatun Ni'mah

Universitas Islam Zainul Hasan Qluluknikmahasa@gmail.com

\section{Edi Kurniawan Farid}

Universitas Islam Zainul Hasan Q edikurniawanfarid@gmail.com

\section{Syamsul Ma'arif}

Universitas Islam Zainul Hasan Q syam96126@gmail.com

\section{Keywords:}

Learning Arabic

Creating Learning Environment Language Environment Islamic Boarding School

\section{Article Information:}

Submitted: 29 May, 2020

Accepted: 13 November, 2020

Approved: 25 November, 2020

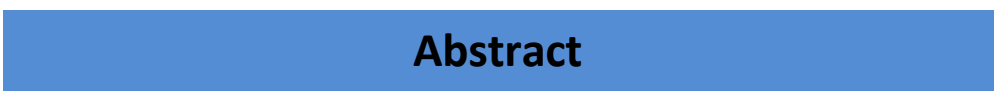

Purpose - This research aims to know the programs for creating the language environment at Al-Mashduqiah Islamic Education Center Patokan Kraksaan Probolinggo, and describe the supporting and the obstacle factors in creating the language environment at AlMashduqiah Islamic Education Center Patokan Kraksaan Probolinggo.

Design/methodology/approach - The researcher uses a qualitative research approach with a case study research design. The techniques that used for collecting data are observation, interview and documentation.

Findings - The result of this research, the programs for creating the language environment at Al-Mashduqiah Islamic Education Center Patokan Kraksaan Probolinggo there are delivering of vocabularies, repeating of vocabularies, language clubs, language court, public speaking, conversation, language month, language competition, and repairing. The Supporting and the obstacle factors in creating the language environment at Al-Mashduqiah Islamic Education Center Patokan Kraksaan Probolinggo consist of internal factors and external factors.

Originality/value - Depend on the results of this research that to creat the language environment needs some efforts such as doing the language programs, practicing, implementing an affective language teaching method, and implementing the language games to reduce boredom and to motivate students in learning foreign language.

Paper type - Research Paper

المقدمة

اللغة لها دور هامّ لحضـارة البشر لأنّ تقدّم الحضيارة لا يمكن وقوعاه إلّا أن يكون هناك اجتهاد

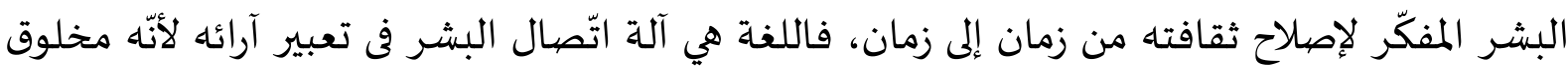
اجتماعي لا يقدر على الحياة فى هذه الدنيا منفردا. والوظيفة الأساسيّة من اللغة هي التعبير عن أفكار ومشاعر وانفعالات الفرد المتكلّم إلى المخاطب. 
اليوم عرفنا أن المعارف تنمو نموّا سريعا واللغة العربيّة هي أوّل اللغة المستخدمة في نموّ المعارف ألماف

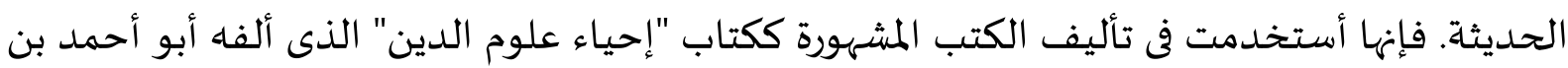

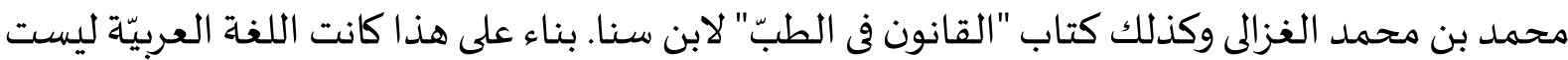

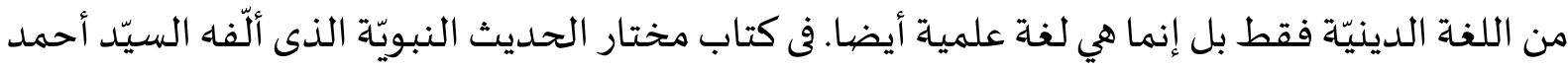

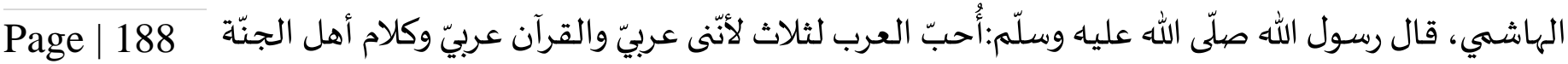
عربيّ (رواه الطبرانى وغيره).(1995 al-Hāshimī).

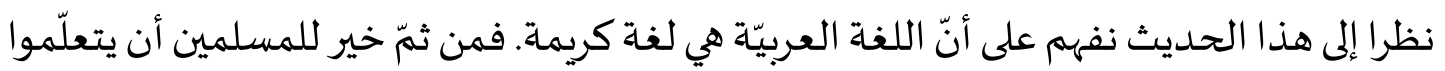

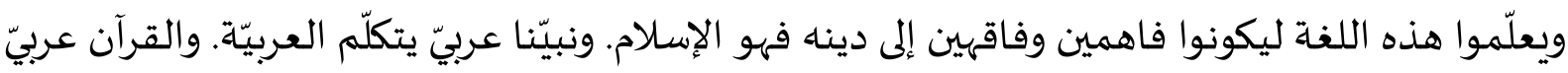

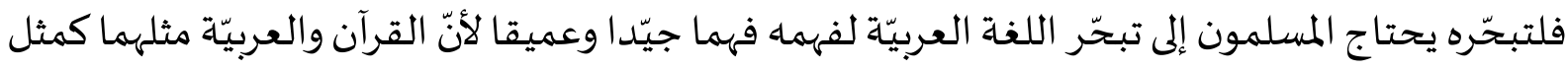

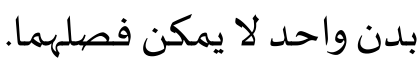

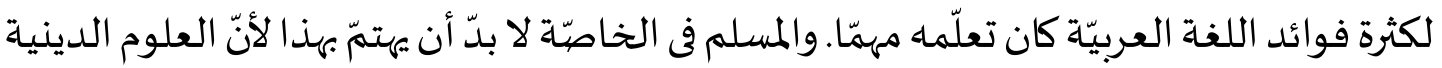

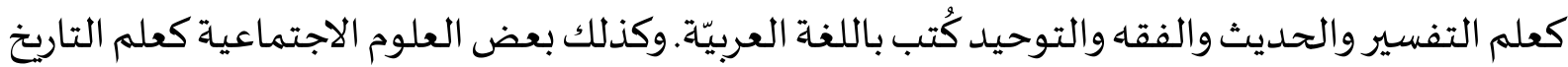

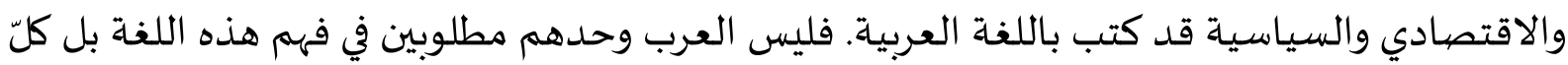

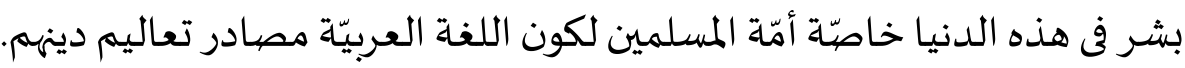

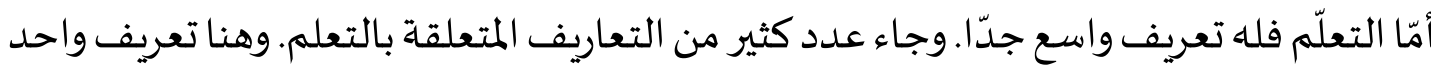

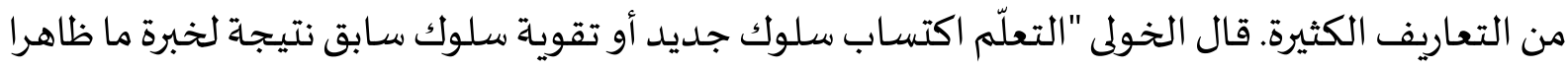

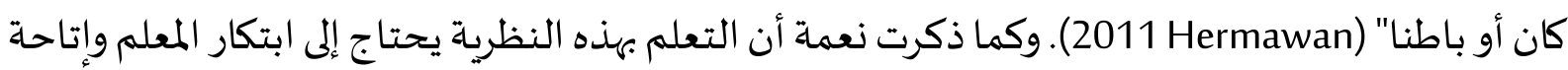

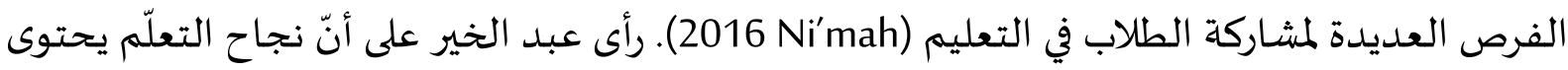

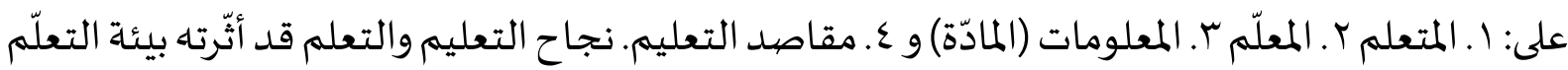

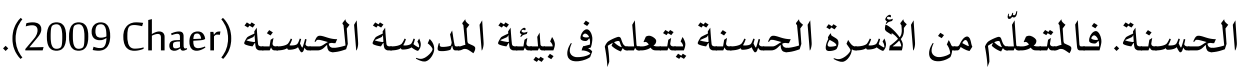

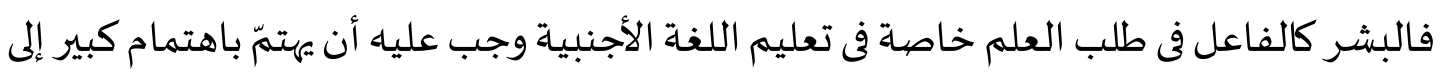

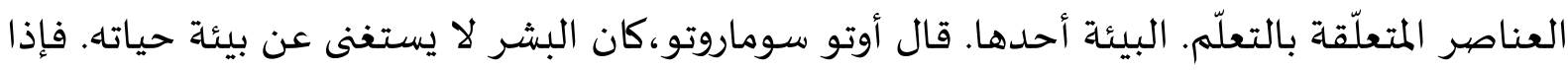

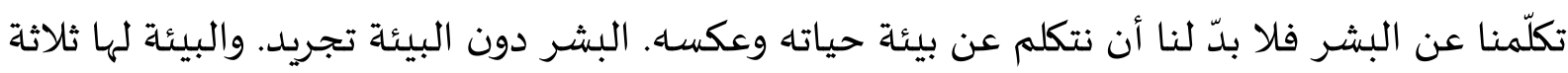

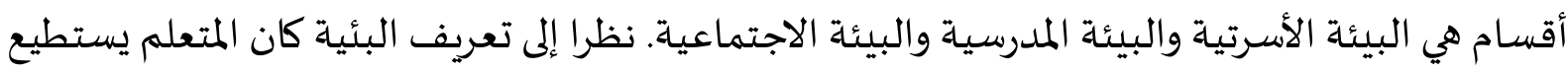

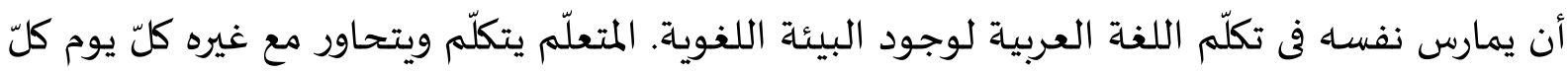

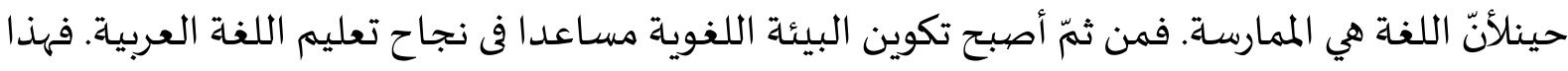

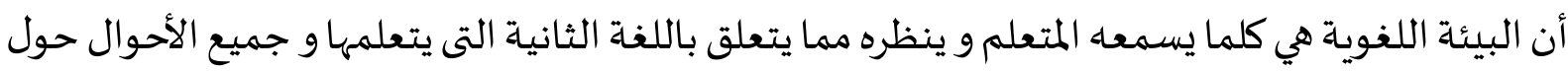

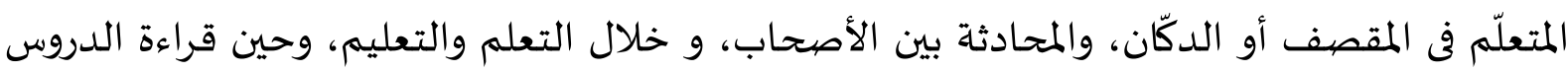

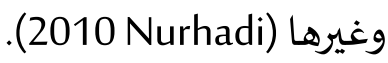

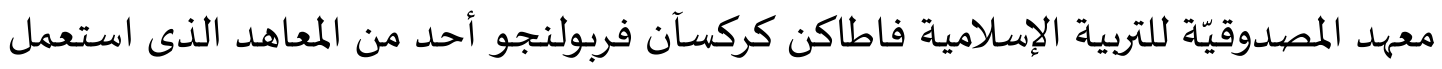

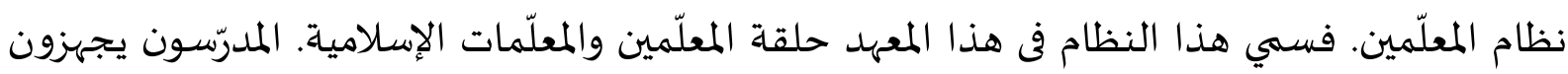


طلّابهم ليقدروا على أن يكونوا معلّمين في أي مكان سكنوا وفى أي عمل عملوا. لهذا المعهد مزايا كثيرة. أحدها اللغة الرسمية التى تكون سببا لمشهوره. فاللغة تاج هذا المعهد. وجب على الطلاب أن يتكلّموا باللغة العربية أسبوعين واللغة الإنجليزية أسبوعين.

لوجود البيئة اللغوية كثير من طلاب معهد المصددوقية يقدرون على تكلمّم اللغة العربية فصيحا و

Page | بعض المتخرّجين من هذا المعهد يستطيعون استمرار دراستهم إلى مصر فى جامعة الأزهار لحسن لغتهم. من 189 هذه الخلفية أراد الباحث معرفة البرامج المتعمدة لتكوين البيئة اللغوية بمعهد المصديدوقياة للتربية الإسلامية فاطاكن كركسآن فربولنجو، ومعرفة العوامل المساعدة والعوامل المعوّقة لتكوين البيئة اللغوية في هذا

\section{أحدث البحث وتميزه}

هناك بحوث أخرى تبحث فيما يتعلق بالبيئة اللغوية منها بحث شمسية في معرفة دور بيئة اللغة لترقية مهارة الكلام في استيعاب اللغة العربية (2015 Syamsiyah). تركز هذا البحث إلى ترقية مهارة الكلام من خلال البيئة اللغوية اعتمادا على النظرية السلوكية، وهذا البحث بحث نظري بينما كان البحث الحالي البحث الميداني من خلال دراسـة الحالة في معهد المصيدوقيّة للتربية الإسلامية فاطاكن كركسآن فربولنجو. والبحث الأخر لمشفعة في معرفة محاولة تكوين البيئة من خلال تطبيق الدروس الإضـافية لترقية مهارة الكلام في المدرسة،و2016 Musyafa'ah) وتركز هذا البحث إلى معرفة تطبيق الدروس الإضافية لتكوين البيئة

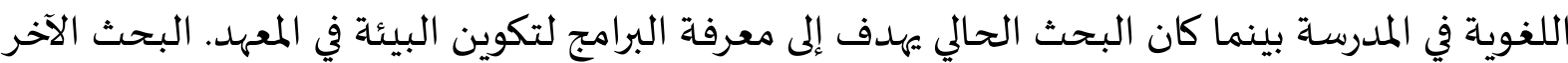
فيما يتعلق بالبيئة اللغوية لمبرورة، وهذا البحث بحث نظري عن البيئة اللغوية العربية من دون التطبيق الميداني (2017 Mabruroh).

\section{طريقة البحث}

استخدم الباحث في هذا البحث المدخل الكيفي بمنهج الدراسـة الحالة. وهي الأسلوب في البحث الوصفي بدراسـة الحالة فردا ما أو جماعة ما أو مؤسسـة ما، كالأسرة أو المدرسـة أو المصنع عن طريق جمع

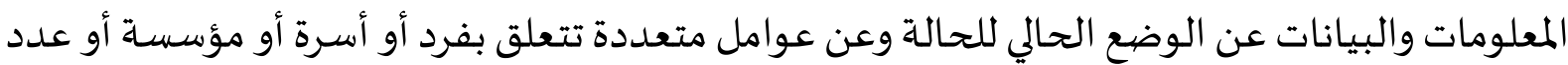
قليل من الأفراد أو نظاما اجتماعيا وحالات محددة (Adas' 2005). وأما الطريقة لجمع البيانات المستخدمة فهي الملاحظة والمقابلة والوثائق.

\section{المناقشة}

البيئة عند رأي محمد جمال هي المؤثرات والامكانات والقوى المحيط بالفرد، والتى يمكنها أن تؤثر على جهوده للحصول على الاستقرار النفسي والبدني في معيشته.(n.d,Maḥfūz) وعند رأي صالح عبد العزيز في كتابه التربية الحديثة هي كل ما يحيط بالإنسان من عوامل الطبيعية أو ظروف بشرية واجتماعية، وليس هناك شكّ أن الإنسـان يؤثر على البيئة وعكسـ.(1974 al-'Azīz) أما البيئة اللغوية كما رأى هيدى دولاى هي كلما يسمعه المتعلم و ينظره مما يتعلق باللغة الثانية التى يتعلمها و جميع الأحوال حول المتعلّم 
في المقصف أو الدكّان، والمحادثة بين الأصحاب، و خلال التعلم والتعليم، وحين قراءة الدروس وغيرها (2010 Nurhadi)

فالبيئة التى يقصدها الباحث هنا فهي البيئة اللغوية المحدودة في اللغة العربية، إذن البيئة هنا جميع الأشياء والعوامل المادية والمعنوية التى لها أثر في عملية التعليم وترغب الطلاب فى ترقية اللغة العربية Page | وتدفعهم وتشجعهم على تطبيقها فى واقع حياتهم اليومية كما رأى مرزوقى، أو هي كلما يسمعاء المتعلم و 190 ينظره من المؤثرات المهيئة والامكانات المحيطة باه التى لها علاقة باللغة العربية التى يتعلمها، والتى بها يظهر جهود الطلاب للحصول على النجاح فى تعلم وتعليم اللغة العربية. أما اللغة عند ابن جنى هي أصوات يعبر بها كل قوم عن أغراضهم.(Iinم Jinn 'Ulyān 1952' 1992) وقال ابن خلدون فى تعريف معنى اللغة: اعلم أن اللغة كلها ملكات شبيهة بالصناعـة إذ هي ملكات في اللسان للعبارة عن المعانى، وجودتها وقصورها بحسب تمام الملكة أو نقصانها وليس ذلك بالنظر إلى المفردات، وانما هو بالنظر إلى التراكيب (Ulyān' ' 1992). وعرف مصطفى الغلاييني بأن اللغة هي: ألفاظ يعبر بها كل قوم عن

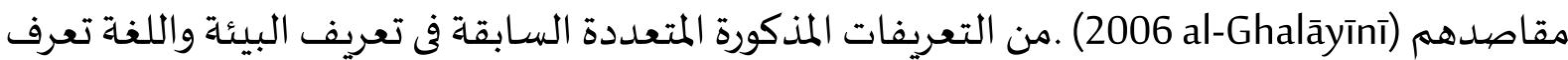
بأن البيئة هي كل شيء حول الناس الذى له أثر عظيم على نشأتهم وتنميتهم. واللغة هي الفاظ التى استخدمها المرء للمعاملة والمعاشرة بينهم ولتعبير عن أغراضههم وآرائهم ومقاصيده. فالبيئة التى بحثها الباحث هنا البيئة اللغوية فهي جميع الأشياء والعوامل الموجودة حولنا المتعلقة بترقياة اللغة وتنميتها وتطويرها. وقال حليمي زهدي هي جميع الأشياء والعوامل المادية والمعنوية التى لها أثر في عملية التعليم وترغب الطلاب فى ترقية اللغة العربية وتدفعهم وتشجعهم على تطبيقها فى واقع حياتهم وكذلك البيئة كلما يسمعاه المتعلم و ينظره من المؤثرات المهيئة والامكانات المحيطة باء التى لها علاقة باللغة

والتى بها يظهر التأثير فى جهود الطلاب للحصول على النجاح فى تعلم اللغة وتعليمها (2009 Zuhdi). البيئة اللغوية هي كل ما سمعت ورأت من المتعلمين المتعلق باللغة التى يدرسونها. كما اقترح Krashen ، هناك نوعان من البيئة اللغوية: البيئة الرسمية وغير الرسمية.(1976 Krashen) البيئة الرسمية: تحتوى على جوانب مختلفة من التعليم الرسمي وغير الرسمي، والأغلب كان التعليم رسميا، في الفصيول الدراسية أو في المختبر. يمكن أن توفر هذه البيئة الرسمية المعرفة للمتعلمين فى شكل خطاب اكتساب اللغة (المهارات

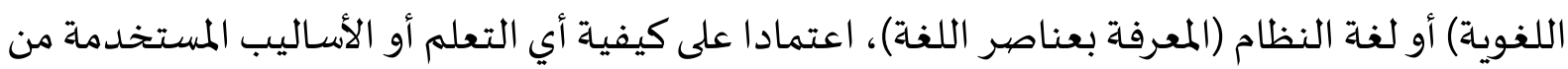
نفس المعلم. لكن في الغالب هناك اتجاه أن هذه البيئة تعطى معرفة نظام اللغة الرسمية أكثر من الخطاب للغذة. البيئة غير الرسمية: فهذه البيئة غير الرسمية قدم لاكتساب لغة الخطاب بطبيعة الحال، وغالبا ما يقع خارج الفصول الدراسية. تكوين هذه البيئة لوسائل الاتصال بالمعلمين، الطلاب، مديري المدرسـة، والموظفين والأشخاص المتورطين فى الأنشطة المدرسية. يرى فؤاد أفندي هناك ثلاثة على الأقل من الأشياء لتكوين البيئة اللغوية، ألا وهي: وجود موقف إيجابي تجاه "اللغة العربية" والتزام قوى للنهوض بتدريس اللغة العربية من الأطراف المعنية. الأطراف ذات صلة المعلمين ( ) الرئيسية r ) معلموا اللغة العربية r) الطلاب ع) ويكون كبيرا إذا كانت عناصر "الموارد 
البشرية" الكامل من المدارس الجيدة تنتج المبين والانضمام إلى التعليم يعتمد عليه. أن وجود هذا الرقم فى مدرسة التى قادرة على التواصل باللغة العربية، سواء كانت أصلية أم لا. وجود تخصيص الأموال الكافية لتوفير الوسائل اللازمة (2009 Effendi).

إن اللغة تنشأ وتتطور على محيطها وبيئتها الاجتماعية وعندما توجد مؤثرات خارجياة يحصل

Page | التفاعل ويؤدى إلى تكوين السلوك اللغوي يشجع المتعلم (2009 Zuhdi). كان تعلم اللغة الأجنبية هو عملية 191 مترددة بأنواع المظاهرة المعضلة فهذه تؤدى إلى وجود عدة من التعريفات المختلفة عند أهلها. وكان التعلم فى اللغة يؤثر على العاملين: العامل الداخلي والعامل الخارجي (2009 Rosyidi). والعامل الداخلي كما قال نانا سوجانا هو العامل فى داخل الطلاب كمهارة نفسـه. وبجانب ذلك الدوافع فى التعلم، الهمة والقدرة، العدة النفسية، الاجتماعية والاقتصيادية، الأحوال الجسمية والروحية هي التى من العوامل الداخلية. وأما العامل الخارجي هي كل ما وقع خارج نفس الطلاب كالمواد الدراسية والوسائل التعليمية أو البيئة التى سكنوا فيها. 2019 Sudjana) العامل الثانى هو الذى يتعلق ببحثنا هذا. البيئة المتعلقة باللغة العربية. العوامل التى فيها التأثير في تعليم الطلاب اللغة يؤيد البيئة اللغوية التى وضعها الأسـاتيذ ووجب أن تكون فيها العوامل المساعدة أكثر من العوامل المعوقة. فإن البيئة اللغوية لها دور كبير لنجاح الطلاب في لئى تعلم اللغة العربية (2009 Zuhdi). Krashen)،تنسم البيئة إلى قسمين: البيئة الاصطناعياة والبيئة غير الاصطناعية (الطبيعية) 1976) ولكلهما دور نلخصه فيما يلي. إن البيئة الاصطناعية هي احدى البيئات اللغوية التى ترتكز على التى سيطرة القواعد أو نظام اللغة في لغة الهدف مع التوعية، والتوعية على قواعد لغة الهدف تمكن إقامتها بالمنهج الاستنتاجي أو المنهج الاستدلالي. والمقصود بالمنهج الاستنتاجي هو أن يشرح المعلم للمتعلم عن قواعد لغة الهدف حتى كان المتعلم فاهما عارفا ومستوعبا على القواعد فيفعله المعلم في التطبيق. وأما المنهج الاستـلالي فهو الحالة لمعرفة الأشكال اللغوية ثم يسوقه المعلم ليكون واجدا نفساه عن تلك القواعد. ويرى إلياس أن أثر التعلم الرسمي (البيئة الاصطناعية) يمكن نظره على ناحيتين؛ ترتيب اكتساب اللغة الثانية والسرعة أو نجاح سيطرة اللغة الهدف (2010 Nurhadi). أما البيئة الطبيعية فيقصد بها استخدام اللغة العربية بقصد التفاهم ونيل المعلومات أي مع التركيز على المحتوى، هذه الحالة اللغوية تجرى طبيعية تدخل فيها ما نفعل حين يتحدث مستخدمو اللغة الأولى أو الأجنبية فى الشـارع أو الملعب مثلا، إنّ التركيز هنا لايكون على الصيخ اللغوية بل على المحتوى أو السياق. البيئة اللغوية لمتعلمها يعنى بها الباحث بلاد العرب، كى يجدها في بلادنا ولولا يسكن فيها العرب. فمن ثم يحتاج إلى تكوين البيئة اللغوية ولوكان من المستحيل أهها تشباء البيئة الأصلية. اشتملت البيئة الطبيعياة هي اللغة التى استخدمها الأصحاب فى نفس المرحلة، اللغة التى استعملها المتعلمون، اللغة المستخدمة في الأخبار المطبوعة واللغة استخدمها الأساتيذ فى عملية التعليم والتعلم فى فصل اللغة وغيره. 
ومن إستراتيجيات لتكوين البيئة اللغوية هي إقامة سكن الطلاب ليكون مركزين فى مكان واحد ويسهل على المشرفين والمدرسين ملاحظتهم وإرشادهم وإقامة أنشطة عربية خارج وقت الدراسة. وتعيين الأماكن المحظورة فياه التحدث والكلام بغير اللغة العربية، المثال: المقصف ومكاتب المدرسـة. وإقامة البرامج العربية فى وقت معين يتصرف الطلاب فيها ميولهم النفسية والفنية من مسرحية وخطابات ومنباريات وهذا

Page | البرامج تحت رعاية وإرشاد مشرفي والمدرسي اللغة العربية. وتدريب الطلاب على إلقاء الخطابة بالعربية في 192 المحاضرة في يوم معين (2009 Zuhdi).

إن المحاولة فى تكوين البيئة اللغوية هي مسؤولية المعهد وأهله كالمؤسسة التربوية الإسلامية، كما عرف أن اللغة تاج المعهد فكل واحد من أهل المعهد من المدرسين والطلبة وكذلك الموظفون مسؤولون عن محافظتها وترقية جودتها. سوف يشعر جميع أعضاء المعهد على المقاصيد والانتفاع فى تكوين البيئة اللغوية ثم يدافع سعيهم وجهدهم على جميع البرامج المتعلقة بالبيئة اللغوية. ومحاولة هيئة تطوير اللغة وقسم إحياء اللغة المركزي الأخرى التى لها دور مهم هي إعطاء العقوبات فى المحكماة اللغوية لمن لم يشارك البرامج

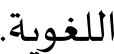

ومن محاولات تكوين البيئة اللغوية هي أن أوجب مدير المعهد على جميع الطلاب والطالبات التكلم

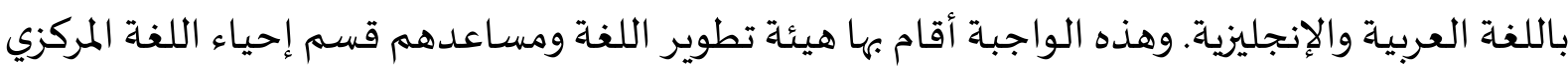
من رابطة الطلبة المعلمين والمعلمات الإسلامية المصدوقياة. ومن البرامج المعتمدة لمساعدة هيئة تطوير اللغة وقسم إحياء اللغة المركزي فف تكوين البيئة اللغوية هي المراقبة فى عملية استخدام اللغة فى المحادثة اليومياة، قراءة كشف الحضيور فى البرامج اللغوية المعينة، تنفيذ تزويد المفردات كل صبباح، تنفيذ تكرير المفردات كل يوم قبل النوم، عقد المحكمة اللغوية، عقد تفتيش كتيب المفردات ومعجم العربي والإنجليزي للطلبة، تنفيذ المحاضرة مرتين فى الأسبوع، تنفيذ المحاورة بين طلاب الجدد والقدماء مرتين في الأسبوع، أمر الطلبة بتكوين الجمل من المفردات الملقية في الأسبوع، عقد المشاورة اللغوية بين هيئة تطوير اللغة وقسم إحياء اللغة المركزي لحل المشكلات اللغوية عند الطلبة، تنفيذ الإشراف على الفرقة اللغوية، إعداد المادة لتزويد المفردات، إعداد المادة للمحاورة، الإشراف والملاحظة على مسؤولي اللغة فى المسكن، عقد الاستماع اللغوي إما أن يكون الاستماع من الغناء أو الأخبار

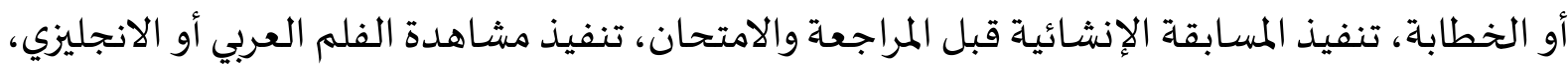
تنفيذ أسبوع اللغة أو شهر اللغة، تعليق كتابة لوحات المفردات في الأمكنة المعينة، تعليق كتابة المصطلحات فى الأمكنة المعينة، عقد المسابقة التمثيلية، عقد الأولمبية اللغوية المصددوقياة،وعقد المبارات الانتخابية لمالك ومالكة وأمير وأميرة اللغة. وقد عرّف هيئة إشراف اللغة وقسم إحياء اللغة المركزي أن الأهداف من المحاولة فى تكوين البيئة اللغوية بمعهد المصيدوقية للتربية الإسلامية هي تعويد الطلبة على التكلم باللغة العبرية أو الإنجليزية. بهذا الهدف المهم في تعليم اللغة الأجنبية في هذا المعهد سوف يكون ناجحا، فالهدف كما ذكر سابقا "تعليما 
Page | الهمة والقدرة، العدة النفسية، الاجتماعية والاقتصادياة، الأحوال الجسمية والروحية هي التى من العوامل

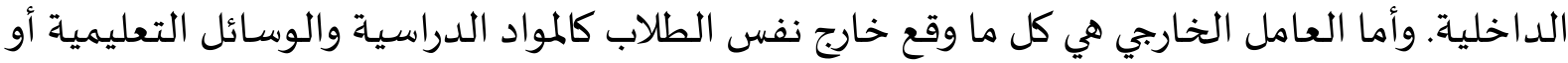
البيئة التى سكنوا فيها (Sudjana) 2019). وإضافة إلى ذلك، حث فريد على أهمية المواد الدراسية التي تلائم

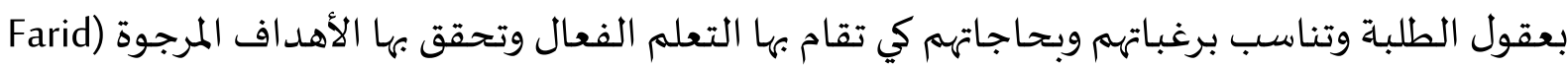

أما العوامل المساعدة التي فيها عوامل داخلية وخارجية في تكوين البيئة اللغوية بمعهد المصددوقية للتربية الإسلامية فهي () وجود الأساتيذ والأستاذات الذين لديهم القدرة الجيدة والكافية في تعليم اللغة

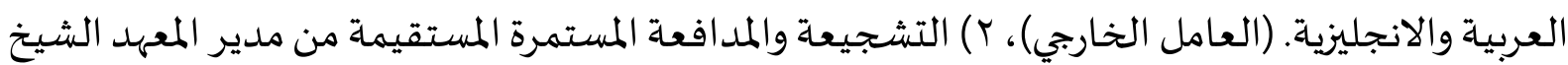
الحاج مخلصين سعد الماجستر لأنه متخرج من معهد الأمين مادورا ومعهد دار السلام كونتور. (العامل

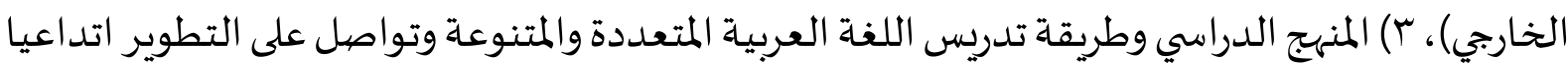

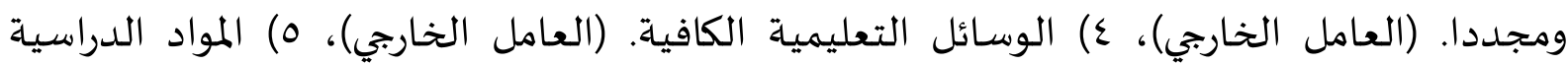

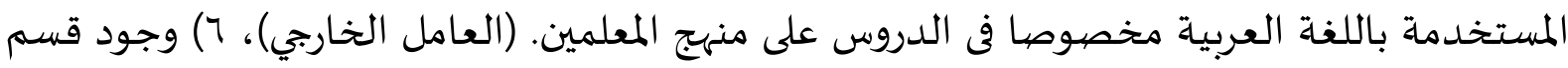

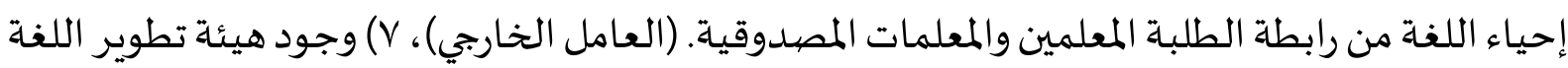

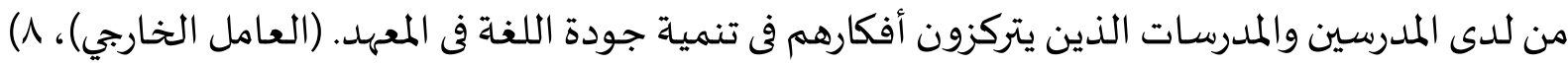
وجود الترتيب ونظام ومحكمة اللغة والتجسس والعقاب للطلاب. (العامل الخارجي)، 9) إعطاء الهدية المداية للطلبة المنجزة في المهارات اللغوية. (العامل الخارجي). أما العوامل المعوقة التي فهها عوامل داخلية وخارجية في تكوين البيئة اللغوية بمعهد المصدوقية الماتية

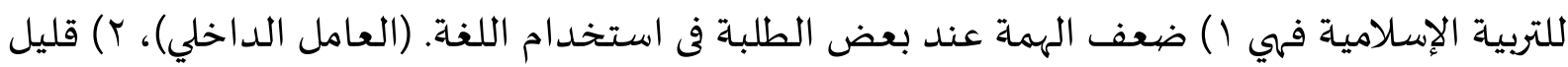

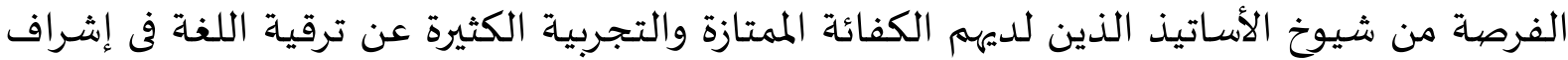

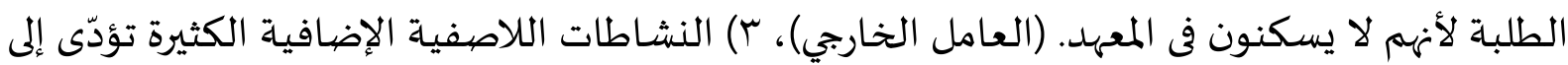

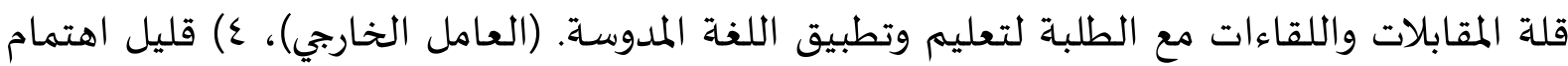
الأساتيذ الجديدة إلى حض الطلبة فى المحادثة بينهم باللغة الرسمية هي العربية والإنجليزية (العامل

وقد عرّف هيئة تطوير اللغة أن من العوامل المعوقة في تكوين البيئة اللغوية الأكبر هو ضعف الهمة

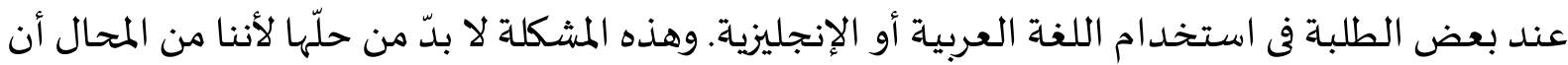

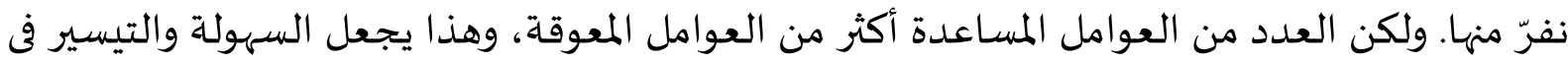


تكوين البيئة اللغوية. والحال مطابق برأي حلىى، أن فى تكوين البيئة اللغوية وجب أن يكون العوامل المساعدة أكثر من العوامل المعوقة (2009 Zuhdi).

\section{الخلاصة}

يتم تكوين البيئة اللغوية بمعهد المصدوقية للتربية الإسلامية فاطاكن كركسآن فربولنجو من

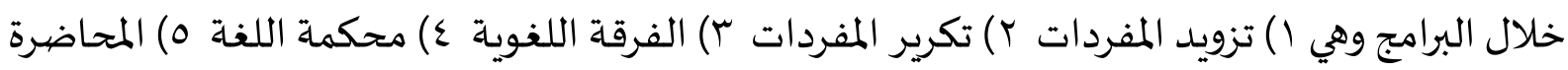

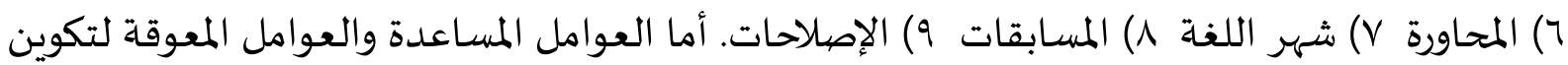
البيئة اللغوية فتتكون من العوامل الداخلية والعوامل الخارجية.

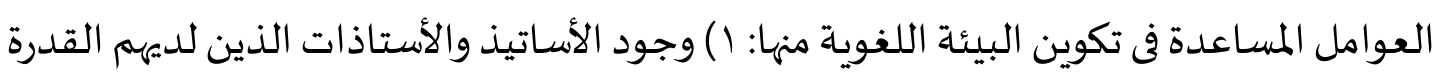

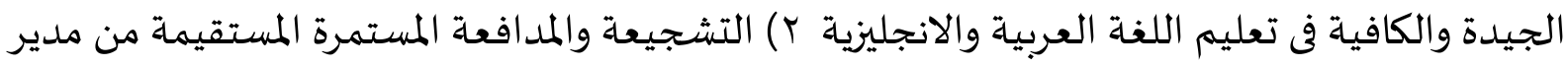
المعهد r) المنهج الدراسي وطريقة تدريس اللغة العربية المتعددة والمتنوعة وتواصل على التطوير اتداعيا

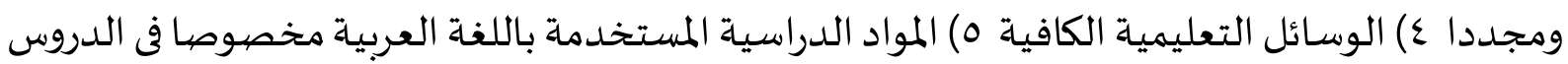

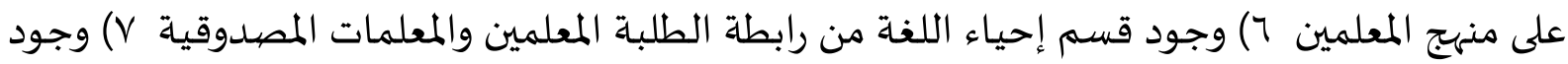

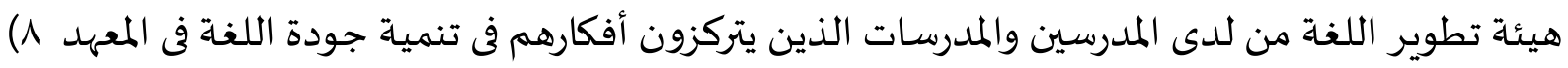
وجود الترتيب ونظام ومحكمة اللغة والتجسس والعقاب للطلاب و 9) إعطاء الهدية للطلبة المنجزة في المهارات اللغوية. أما العوامل المعوقة في تكوين البيئة اللغوية منها: ( ) ضعف الهمة عند بعض الطلبة في استخدام

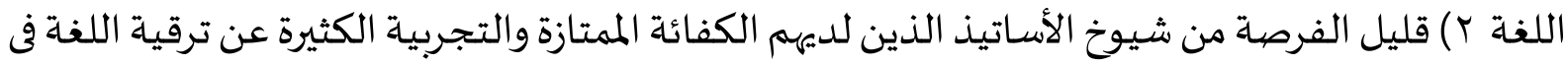

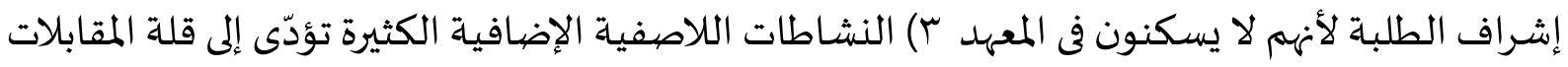

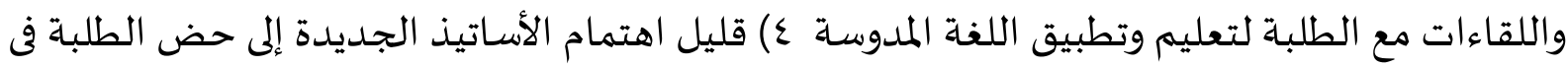
المحادثة بينهم باللغة الرسمية هي العربية والإنجليزية.

\section{اللاستفادة}

يجب أن يكون هناك سعي واضح فيما يتعلق بالمحاولات في تكوين البيئة اللغوية. وجود

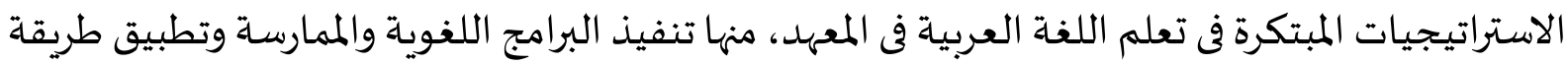
التعليم اللغويي الفعال وتطبيق الألعاب اللغوية لتخفيف المللل ولمدافعة رغبة الطلبة في تعليم اللغة التهائ

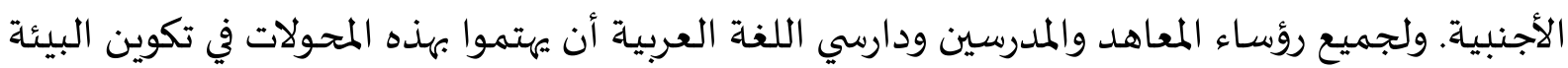
اللغوية.

\section{المراجع}

al-Hāshimī, Aḥmad .1990. Mukhtār al-Hadīth. Translated by Zaini Mahmud. Jakarta: Pustaka

Amani.

Al-'Azīz ,Șaliḥ 'Abd. I १V乏 .al-Tarbiyah al-Ḥadīthah :Māddatuhā - Mabādiuhā -Tatbīqātuhā 
al-'Amaliyah .Muassasat al-Ma'ārif li a-Ṭibā'ah wa al-Nashr.

Jinnī , Abī al-Fatḥ 'Uthmān Ibn. 190r . al-Khașāiș J. 1. Beirut : Dār al-Kutub al-'Arabī.

'Adas ،'Abd al-Raḥmān .r. . o . al-Bahth al-'Ilmī (Mafhūmuhū, Adawātuhū, Asālībuhū). Al-

Riyāọ : Dār Usāmah li al-Nashr wa al-Tawzī'.

'Ulyān ,Aḥmad Fuād . 19१ץ .al-Mahārāt al-Lughawiyah; Māhiyatuhā wa Tarīqatu Tadrīsihāa . al-Riyāọ : Dār al-Muslim.

Al-Ghalāyīnī , Mușțafā .r. . . . Jāmi' al-Durūs al-'Arabiyah .Beirut :Dār al-Kutub al-'Ilmiyah.

Chaer, Abdul. 2009. Psikolinguistik Kajian Teoritik. Jakarta: PT. Rineka Cipta.

Effendi, Ahmad Fuad. 2009. Metodologi Pembelajaran Bahasa Arab. malang: misykat.

Farid, Edi Kurniawan. 2018. "Tațwīr al-Mawād al-Ta'līmiyah li Mahārah al-Istimā' li Dāris alLughah al-'Arabiyah li Ghayri al-Nāțiqīn Bihā." ALSUNA: JOURNAL OF ARABIC AND ENGLISH LANGUAGE 1 (2):114-20.

https://doi.org/https://doi.org/10.31538/alsuna.v1i2.79.

Hermawan, Ajib. 2011. Metode Pembelajaran Bahasa Arab. Bandung: PT. Remaja Rosda Karya.

Krashen, Stephen D. 1976. "Formal and Informal Linguistc Environments in Language Acquisition and Language Learning." TESOL Quartely 10 (2).

Mabruroh. 2017. “al-Bīah al-Lughawiyah al-'Arabiyah fí Ta'līm al-Lughah al-'Arabiyah li Ghayri al-Nāțiqīn Bihā." Jurnal Alfazuna : Jurnal Pembelajaran Bahasa Arab Dan Kebahasaaraban 2 (1):28-41.

https://doi.org/https://doi.org/10.15642/alfazuna.v2i1.245.

Musyafa'ah, Nurul. 2016. "Muḥāwalah fī Takwīn al-Bīah al-'Arabiyah li Tarqiyah Mahārah alKalām min Khilāl al-Durūs al-Iḍāfiyah fĩ Madrasah "Abū Darrin" al-Thānawiyah KendalDander-Bojonegoro." Al-Ta'rib : Jurnal Ilmiah Program Studi Pendidikan Bahasa Arab 
IAIN Palangka Raya 4 (1):28-41.

https://doi.org/https://doi.org/10.23971/altarib.v4i1.559.

Ni'mah, Mamluatun. 2016. “Memahami Konsep Dasar Teori Bahasa Dan Pembelajaran

https://ejournal.inzah.ac.id/index.php/attalim/article/view/181.

Nurhadi. 2010. Dimensi-Dimensi Dalam Belajar Bahasa Kedua. Bandung: sinar baru algesindo.

Rosyidi, Abd. Wahad. 2009. Media Pembelajaran Bahasa Arab. malang: uin press.

Sudjana, Nana. 2019. Dasar-Dasar Proses Belajar Mengajar. 15thed. Bandung: sinar baru algesindo.

Syamsiyah, Nur. 2015. “Dawru Bīah al-Lughah li Tarqiyah Mahārah al-Kalām fī Istī'āb alLughah al-'Arabiyah" 1 (2):177-85.

http://jurnal.uinsu.ac.id/index.php/ihya/issue/view/44.

Zuhdi, Halimi. 2009. al-Bīah al-Lughawiyah Takwīnuhā wa Dawruhā fĩ Iktisāb al-'Arabī. Malang: Uin Press. 